\title{
MR-Eye: High-Resolution Microscopy Coil MRI for the Assessment of the Orbit and Periorbital Structures, Part 1: Technique and Anatomy
}

\author{
(D) N.W. Dobbs, (D).J. Budak, (D) R.D. White, and (DI.A. Zealley
}

\begin{abstract}
SUMMARY: Microscopy coil MR imaging of the orbits has been described previously as a technique for anatomic depiction. In the first part of this 2-part series, the improvement in spatial resolution that the technique offers compared with conventional MR imaging of the orbits is demonstrated. We provide a guide to implementing the technique, sharing pearls and pitfalls gleaned from our own practice to make implementation of microscopy coil MR imaging at your own center easy. As a quick reference guide to the small-scale structures encountered when reading the studies, a short anatomy section is included, which doubles as a showcase for the high-quality imaging that can be obtained. In the second part, our experience of microscopy coil MR imaging in day-to-day clinical practice takes it far beyond being a useful anatomic educational tool. Through a series of interesting cases, we highlight the added benefit of microscopy coil MR imaging compared with standard orbital MR imaging.
\end{abstract}

ABBREVIATION: MC-MRI = microscopy coil MR imaging

$I^{n+m}$ maging of orbital and periorbital structures and pathologies presents challenges due to the various limitations intrinsic to ultrasound, CT, and conventional MR imaging. ${ }^{1,2}$ Management of structural pathology in and around the orbit is guided by knowledge of the compartments involved and the tissue of origin. ${ }^{3}$ Both the initial surgical approach ${ }^{4}$ and subsequent reconstruction techniques are influenced by factors that can only be resolved at a very small scale, sometimes at submillimeter resolution. Changes of this order cannot be resolved using CT or conventional head coil MR imaging, while ultrasound is limited to demonstration of soft tissues only and cannot demonstrate relationships with bony structures.

The use of microscopy coil MR imaging (MC-MR imaging) to depict orbital anatomy has previously been reported, generally for research studies. ${ }^{5-7}$ In this 2-part article, we first aimed to equip the reader with an understanding of MC-MR imaging techniques to enable implementing this simple, straightforward imaging at his or her own institution. We also aimed to refresh and

Received July 18, 2019; accepted after revision January 21, 2020

From the Department of Clinical Neuroscience (N.W.D.), Western General Hospital, Edinburgh, United Kingdom; Qscan Radiology Clinics (M.J.B.), Gold Coast, Australia; Department of Clinical Radiology (R.D.W.), University Hospital of Wales, Cardiff, United Kingdom; and Department of Clinical Radiology (I.A.Z.), Ninewells Hospital, Dundee, United Kingdom.

Please address correspondence to Nicholas W. Dobbs, MBBS, Department of Clinical Neuroscience, Western General Hospital, Edinburgh, United Kingom; e-mail: nicholas.dobbs@nhs.net

- Indicates open access to non-subscribers at www.ajnr.org

http://dx.doi.org/10.3174/ajnr.A6495 expand the reader's knowledge of orbital anatomy, essential for interpretation of MC-MR images. Pearls and pitfalls of the technique that we have gleaned from everyday practice are shared, to make implementation of the technique easier.

In Part 2, in addition to using MC-MR imaging as a tool for anatomic depiction, we explore the benefits of using MC-MR imaging in everyday clinical practice. We have previously described the application of MC-MR imaging in preoperative planning for Mohs micrographic surgery for nasofacial skin neoplasms. ${ }^{2}$ Collaboration with ophthalmic surgeons has extended our practice to provide high-resolution imaging of the orbits and surrounding structures.

Figure 1 demonstrates the striking difference in image resolution between conventional head coil orbital MR imaging and MC-MR imaging. The high spatial resolution offered means that the ophthalmic surgeons at our institution believe that they can better plan surgical procedures and preserve structures that are difficult or impossible to reconstruct, while at the same time maximizing the accuracy of resection margins.

\section{Technique}

By means of a small radiofrequency receiver coil placed in close proximity to the orbit structures, a voxel size of $300 \mu \mathrm{m}$ can be resolved with high SNR. This high SNR is maintained down to the orbital apex, beyond which signal loss becomes an issue and supplementary head coil imaging may be required. As we have previously described for the imaging of nasofacial lesions, ${ }^{2}$ we 


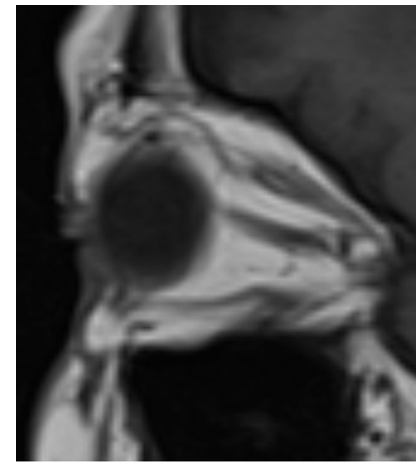

A

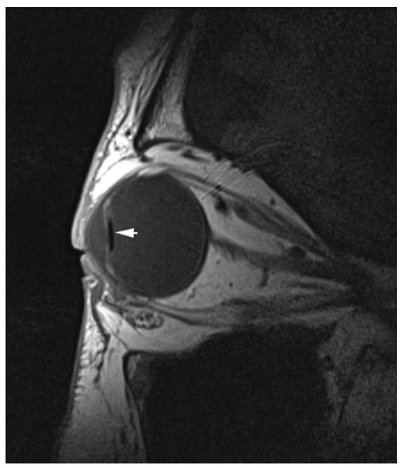

B

FIG 1. The difference between conventional T7-weighted sagittal imaging with a head coil $(A)$ and MC-MR imaging T1-weighted sagittal imaging $(B)$. In this patient, a lens prosthesis (white arrow) inserted during cataract surgery is demonstrated.

\section{Sequence parameters}

\begin{tabular}{lcc}
\hline \multicolumn{3}{c}{ Parameters for MC-MR Imaging } \\
\hline \multicolumn{1}{c}{ Parameter } & T1-Weighted & T2-Weighted \\
TSE & TSE \\
\hline TR (ms) & 400 & 4000 \\
TE (ms) & 15 & 123 \\
Signals averaged & 2 & 3 \\
FOV (mm) & $80 \times 70$ & $80 \times 70$ \\
Martrix & $224 \times 256$ & $224 \times 256$ \\
Section thickness (mm) & 1 & 1.5 \\
Turbo factor & 3 & 13 \\
Voxel size (mm) & $0.3 \times 0.3 \times 1.5$ & $0.3 \times 0.3 \times 1.5$ \\
Acquisition time & $5 \mathrm{~min}, 42 \mathrm{sec}$ & $7 \mathrm{~min}, 6 \mathrm{sec}$ \\
\hline
\end{tabular}

use a 40-mm-internal-diameter small-loop radiofrequency receiver coil with a $1.5 \mathrm{~T}$ MR imaging unit (Magnetom Avanto; Siemens, Erlangen, Germany). This has 32 receiver channels and SQ-engine gradients (maximum gradient field strength, $45 \mathrm{mT} / \mathrm{m}$; slew rate, $200 \mathrm{~T} / \mathrm{m} / \mathrm{s}$ ). Sequence parameters are described in the Table.

Our standard imaging consists of optimized T1-weighted and T2-weighted TSE sequences with $0.3 \times 0.3 \mathrm{~mm}$ pixel size and $1.5-\mathrm{mm}$ section thickness acquired with no intersection gaps. Images can be acquired in any plane depending on the structures to be assessed. We have found that axial acquisitions are optimal to demonstrate the nasal bone, tarsal plate, optic nerve, and the medial and lateral rectus muscles. Sagittal acquisitions are useful for depiction of the orbital septum and the levator apparatus, as well as the superior and inferior rectus muscles. Coronal acquisitions provide an excellent overview of the extraocular muscles, the optic nerve, and any intraconal lesion extension. The presence of orbital fat around most structures means that, generally speaking, T1-weighted imaging delivers the best depiction of structural involvement, with T2-weighted and occasionally T1-weighted fat-saturation sequences used to aid lesion characterization if this is required.

With T1-weighted acquisitions taking $>5$ minutes, and T2weighted acquisitions, $>7$ minutes, movement needs to be minimized to make the best use of the high SNR to deliver high-resolution imaging. Thus, while we make every effort to ensure

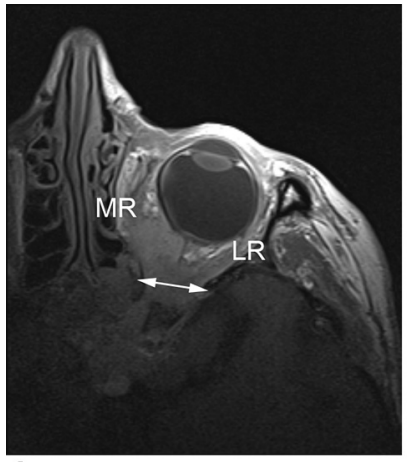

A

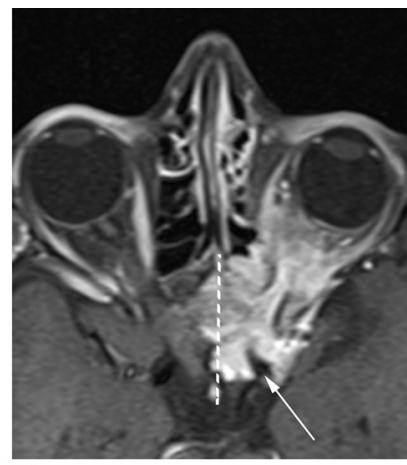

B

FIG 2. $A, M C-M R$ imaging T1-weighted axial image demonstrates a sphenoid wing meningioma, engulfing the optic nerve as well as the medial (MR) and lateral rectus (LR) muscles. MC-MR imaging demonstrates widening of the superior orbital fissure (double-headed arrow), but beyond the orbital apex, the signal-to-noise ratio is low. $B$, Head coil T7-weighted fat-saturation postcontrast axial image demonstrates the lesion surrounding the left internal carotid artery (white arrow), infiltrating the left cavernous sinus and extending past the midline (dotted white line) in the intercavernous sinus.
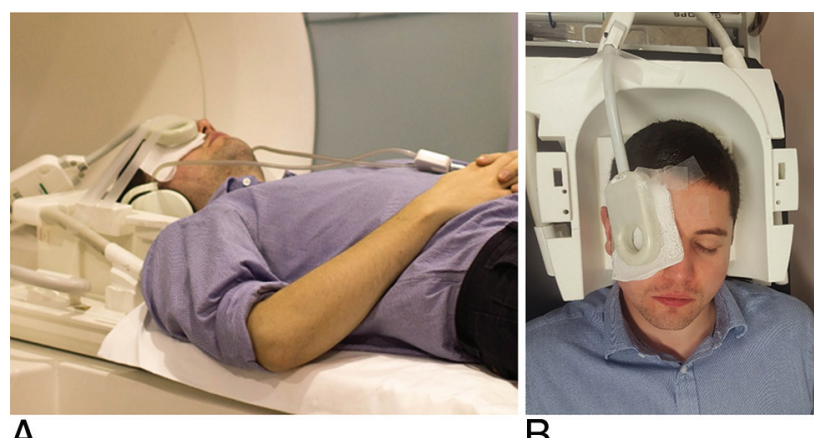

B

FIG 3. Correct patient and coil positioning. Photographs of the author (N.W.D.) showing final patient and coil positioning in the imaging magnet with headphones wedged against head coil for immobilization $(A)$ and coil positioning over the orbit with extensive taping $(B)$.

patient comfort, robust immobilization, essential for good-quality MC-MR imaging, is applied surreptitiously and with great care. The patient is positioned head first in the scanner bore with the head in the posterior portion of a head coil. In addition to offering some immobilization, this framework also allows easy transition to standard head coil imaging if required for lesions extending deep to the orbital apex (Fig 2).

Headphones are used both to help relax the patient and to minimize lateral head movement by having the headphones positioned flush against the head coil. Once the head is immobilized, gauze pads are placed over the orbit for comfort and the receiver coil is taped firmly in place on top of the gauze pads (Fig 3). When we first developed our MC-MR imaging technique for the orbits, we also used the anterior portion of the head coil to further immobilize the patient. However, doing this led to excessive eye movement, compromising image quality.

Despite the long acquisition times, unavoidable small involuntary eye movements appear to have relatively little impact on the image quality. When movement artifacts do become an 


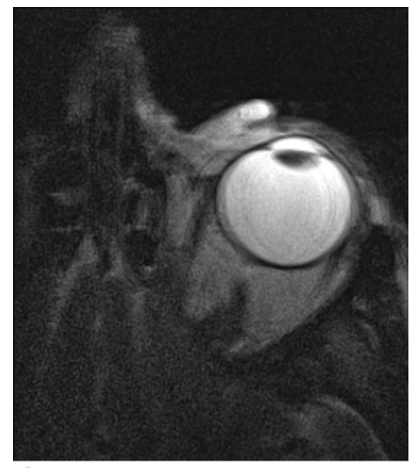

A

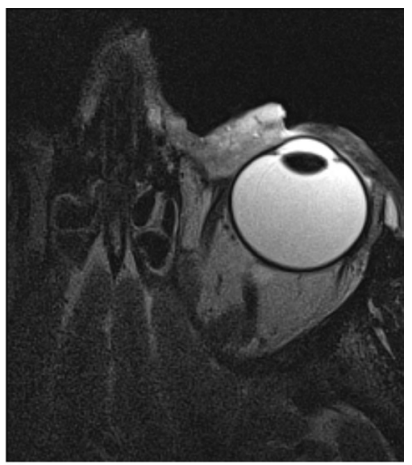

B

FIG 4. Persistence pays off with movement artifacts. A, T2-weighted axial image of the orbit with image degradation due to movement artifacts. $B$, The same patient and same imaging protocol, after ensuring patient comfort.

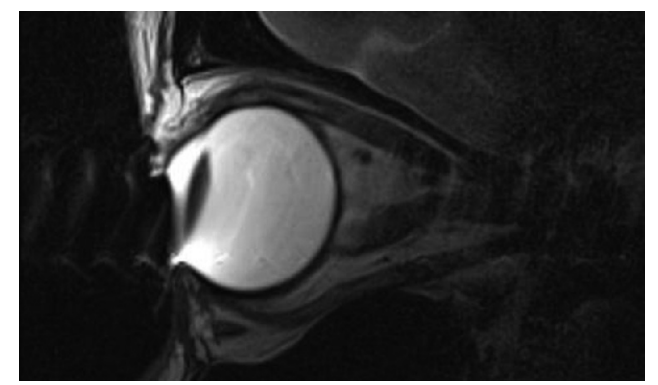

FIG 5. MC-MR imaging T2-weighted sagittal image degraded by metallic artifacts from mascara.
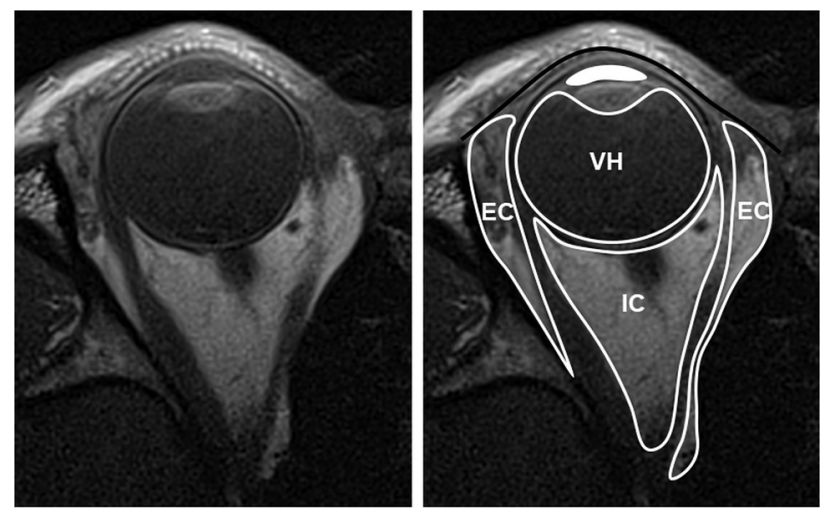

FIG 6. Compartmental anatomy shown by MC-MR imaging TTweighted axial image, original on the left and annotated on the right. The solid black line indicates the orbital septum, defined by native high signal of the tarsal plate; EC, extraconal space, external to the extraocular muscles; IC, intraconal space, inside the extraocular muscles; $\mathrm{VH}$, vitreous humor, behind the lens; solid white fill, aqueous humor, anterior to the lens and ciliary muscles.

issue, taking the time to assess any causative factor or patient discomfort is often effective (Fig 4). Most important, no cosmetics should be worn due to the artifacts arising from metallic elements in these products, particularly mascara (Fig 5).

The capital outlay required to purchase a small-loop receiver coil is small, and at Ninewells Hospital, the coil was

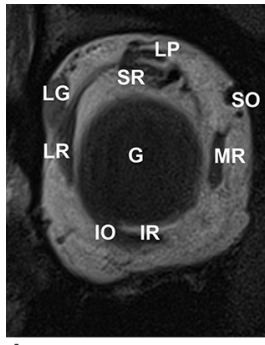

A

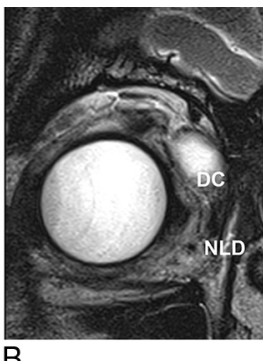

B

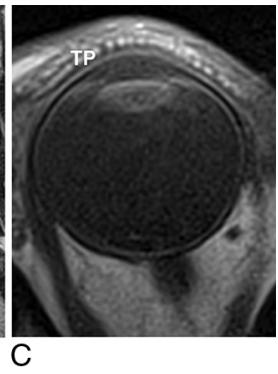

FIG 7. A, T1-weighted coronal MC-MR image. LG indicates the lacrimal gland, positioned superolaterally in the orbit, lying directly inferior to the orbital rim; LP, levator palpebrae superioris muscle; SR, superior rectus muscle; SO, superior oblique tendon; MR, medial rectus muscle; IR, inferior rectus muscle; IO, inferior oblique muscle; LR, lateral rectus muscle; $\mathrm{G}$, globe. $B$, T2-weighted coronal MC-MR image. In this patient, a protruding dermoid cyst (DC) caused epiphora, filling the nasolacrimal duct (NLD) and allowing its demonstration with high signal on T2-weighted imaging. C, T1-weighted axial MC-MR image illustrates the high-signal dots of the meibomian glands within the tarsal plate (TP).

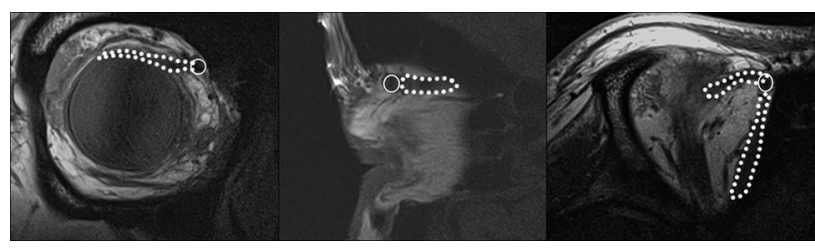

FIG 8. T1-weighted MC-MR images in the coronal, sagittal, and axial planes demonstrating the course of the superior oblique muscle and tendon (dotted line) through the trochlea (circle).

found unused on a shelf. The case-by-case cost is also low, with a scanner time for a 3 -sequence examination of $<20$ minutes. We have found it best to use MC-MR imaging for the imaging of orbital lesions; through its high spatial and contrast resolution, MC-MR imaging can assess the relationship of a lesion to normal anatomic structures in detail sufficient to more confidently guide an appropriate clinical and surgical approach. However, the signal-to-noise ratio beyond the orbital apex is low (Fig 2). For lesions that extend beyond this point, MC-MR imaging can be used as an optional adjunct to traditional imaging if the extra-anatomic information provided stands to influence surgical options.

\section{Orbital Anatomy}

A detailed understanding of orbital anatomy is essential for describing the salient features that the ophthalmic surgeon requires for diagnosis, determining the extent of disease, and preoperative planning. MC-MR imaging allows depiction of this anatomy, from basic compartmental anatomy (Fig 6), muscular anatomy (Fig 7A) including the complex course of the superior oblique muscle (Fig 8), and beyond.

Vision relies on a smooth refractive surface maintained by the ocular surface system-a triple-layer tear film. ${ }^{8,9}$ The most superficial is an oily lipid layer, produced by the meibomian glands of the tarsal plate, which reduce evaporation from the aqueous layer below, which is produced by the lacrimal gland (Fig 7). ${ }^{8}$ Due to 


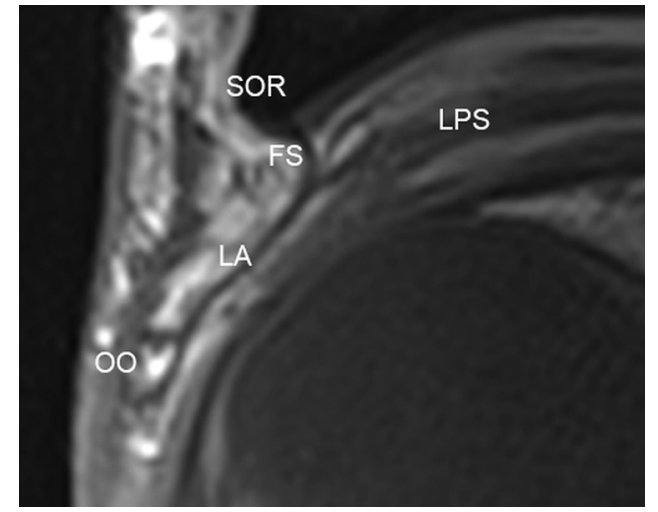

FIG 9. The levator aponeurosis shown on MC-MR imaging T7weighted sagittal image. SOR indicates superior orbital rim; LPS, levator palpebrae superioris; FS, fibrous orbital septum; LA, levator aponeurosis; $\mathrm{OO}$, orbicularis oculi.

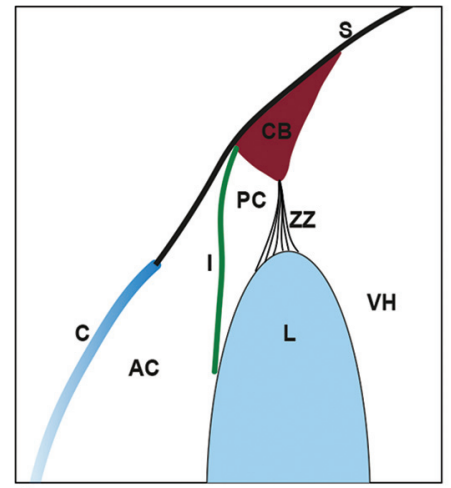

A

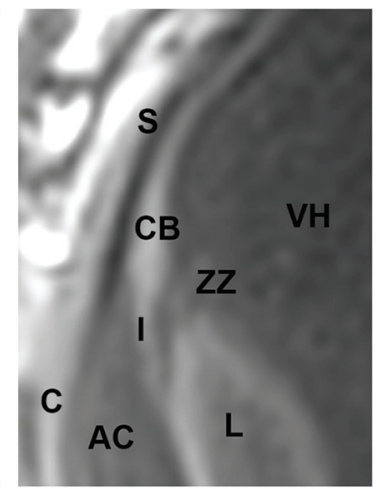

B
FIG 10. Diagrammatic sagittal representation of anterior segment anatomy $(A)$ and its depiction on T-weighted sagittal MC-MR imaging (B). S indicates sclera; $\mathrm{CB}$, ciliary body; ZZ, zonule of Zinn; $\mathrm{VH}$, vitreous humor; I, Iris; AC, anterior chamber; PC, posterior chamber; L, lens; C, cornea.

the lipid content, the tarsal plate is clearly depicted on T1 imaging (Fig 7), allowing localization of pre- and postseptal abnormalities.

The eyelids themselves provide protection from mechanical damage. The elevation and closure of the eyelids are undertaken by the suspensory connective tissue system of the orbit. Primarily, this consists of the levator palpebrae superioris, which traverses the orbit, in conjunction with the superior rectus muscle, before terminating as the levator aponeurosis (Fig 9)

The ring-shaped ciliary body is composed of the ciliary muscle and the ciliary epithelium. Anteriorly, the ciliary body is attached to the sclera, with the iris attaching at the anterior junction of the sclera with the ciliary body. At its most medial extent, the ciliary muscle is attached to the lens via the fibers of the zonule of Zinn (Fig 10). ${ }^{10}$ With variable relaxation or contraction of the ciliary muscle, the zonule of Zinn alters the convexity of the lens and subsequently changes the focal point of the eye. ${ }^{11,12}$

\section{Summary}

The high signal-to-noise ratio of orbital MC-MR imaging facilitates high-resolution imaging, which delineates the small-scale anatomy found in and around the orbits in much greater detail than conventional MR imaging. We have described simple acquisition sequence parameters and discussed practical insights gained from our practice to aid implementation in other centers.

MC-MR imaging of the orbits and periorbital structures is straightforward and requires only a small dedicated surface coil in addition to conventional MR imaging unit equipment, keeping imaging costs low. The high-resolution imaging demonstrated has all been acquired on a $1.5 \mathrm{~T}$ imaging unit, but the technique should apply equally to both less powerful units and, potentially more interesting, to imaging units with a field strength of $\geq 3 \mathrm{~T}$.

MC-MR imaging is most efficiently used as stand-alone imaging for lesions confined to the orbit, to help guide the best management and appropriate surgical approach. For lesions that extend beyond the orbital apex, MC-MR imaging can be used in conjunction with conventional MR imaging, which can lengthen the overall examination time but may still provide useful additional information.

In Part 2 a subsequent article, we describe MC-MR imaging findings for a range of common and less common orbital and periorbital pathologies, highlighting the value of MC-MR imaging for diagnosis, delineation of disease extent, and surgical planning.

Disclosures: Ian A. Zealley-UNRELATED: Employment: National Health Service Tayside, Comments: This is the health service that employs me to perform clinical duties; none directly or indirectly related to this article; Expert Testimony: legal firms, Comments: payment for providing expert witness statements unrelated to this article; Travel/Accommodations/Meeting Expenses Unrelated to Activities Listed: various medical education meetings, Comments: support from medical meeting organizers to present lectures on various topics unrelated to this article.

\section{REFERENCES}

1. Georgouli T, James T, Tanner S, et al. High-resolution microscopy coil MR-Eye. Eye (Lond) 2008;22:994-96 CrossRef Medline

2. Budak MJ, Weir-McCall JR, Yeap PM, et al. High-resolution microscopy-coil MR imaging of skin tumors: techniques and novel clinical applications. Radiographics 2015;35:1077-90 CrossRef Medline

3. Tailor TD, Gupta D, Dalley RW, et al. Orbital neoplasms in adults: clinical, radiologic, and pathologic review. Radiographics 2013;33:1739-58 CrossRef Medline

4. Paluzzi A, Gardner PA, Fernandez-Miranda JC, et al. "Round-theclock" surgical access to the orbit. J Neurol Surg B Skull Base 2015;76:12-24 CrossRef Medline

5. Hoffmann KT, Hosten N, Lemke AJ, et al. Septum orbitale: high-resolution MR in orbital anatomy. AJNR Am J Neuroradiol 1998;19:9194 Medline

6. Kau HC, Tsai CC, Ortube MC, et al. High-resolution magnetic resonance imaging of the extraocular muscles and nerves demonstrates various etiologies of third nerve palsy. Am J Ophthalmol 2007;143:28087 CrossRef Medline

7. Clark RA, Demer JL. Magnetic resonance imaging of the globe-tendon interface for extraocular muscles: is there an "arc of contact"? Am J Ophthalmol 2018;194:170-81 CrossRef Medline

8. Gipson IK. The ocular surface: the challenge to enable and protect vision: the Friedenwald lecture. Invest Ophthalmol Vis Sci 2007;48:4390; 4391-98 CrossRef Medline

9. Govindarajan B, Gipson IK. Membrane-tethered mucins have multiple functions on the ocular surface. Exp Eye Res 2010;90:655-63 CrossRef Medline

10. Delamere NA. Ciliary body and ciliary epithelium. Adv Organ Biol 2005;10:127-48 CrossRef Medline

11. Perumal N, Manicam C, Steinicke M, et al. Characterization of the human aqueous humour proteome: a comparison of the genders. PLoS One 2017;12:e0172481 CrossRef Medline

12. Goel M, Picciani RG, Lee RK, et al. Aqueous humor dynamics: a review. Open Ophthalmol J 2010;4:52-59 CrossRef Medline 